\title{
Control not quantum gate on the states of photon orbital angular momentum
}

\author{
Sergey Andrianov ${ }^{1, *}$, Aleksey Kalachev ${ }^{2}$, Oleg Shindyaev ${ }^{1}$, and Andrey Shkalikov ${ }^{2}$ \\ ${ }^{1}$ Institute for Applied Research, Tatarstan Republic Academy of Sciences, 420111 Kazan, Russia \\ ${ }^{2}$ Zavoisky Physical-Technical Institute, FRC Kazan Scientific Center of RAS, 420029 Kazan, Russia
}

\begin{abstract}
The possibility of effectively creating quantum gates based on orbital angular momentum photon qubits using a Kerr nonlinear medium in a cavity is studied. It is shown how a quantum C-NOT gate can be produced with such qubits through four-wave mixing process. A theory of this gate operation is constructed using an input-output formalism. Parametric matching conditions are obtained for effective gate operation.
\end{abstract}

\section{Introduction}

Development of scalable quantum computers is a cornerstone of modern data processing and automatic control technologies $[1,2]$. The solution of this problem will lead to an unprecedented increase in the performance of computing devices, which is of fundamental importance for creating new materials and medicines, processing of large volumes of data, and optimizing complicated systems. The sufficient progress in this direction was achieved using linear optic quantum computers, in which single-photon states of light act as qubits and quantum logical operation are probabilistic [3, 4]. Such quantum gates can be made deterministic by using nonlinear optical phenomena that occur in, e.g., media with Kerr nonlinearity [5]. The scheme of quantum computer based on polarization photon qubits that used Kerr nonlinearity in a four-wave mixing regime was proposed in paper [6]. The possibility for increasing of two-qubit quantum gates efficiency using optical cavity in such a computer was analyzed in paper [7] on the basis of input-output formalism [8] in its optimum regimes of operation were determined.

\section{Control not gate}

Here, we consider control not gates on four-wave mixing of photons with orbital angular momentum encoding of qubits. We analyze realization of these gates in resonator supporting two basic orbital angular momentum photon modes one of which relates to control qubit and another one relates to target qubit due to their selection by orbital angular momentum filter $[9,10]$. Resonant conditions for the conversion of selected target qubit mode into the second mode of target qubit deviating from the first one are realized with the use of spiral phase plate $[11,12]$ inside resonator. Conditions of parameter matching that ensure effective operation of

\footnotetext{
* Corresponding author: andrianovsn@ mail.ru
} 
the gate were obtained. Supplementing the proposed scheme for a control not gate with the schemes of single-qubit operations for photon qubits based on phase shift elements, beam dividers, and spiral phase plate for orbital angular momentum variation yields the universal set gates necessary for realization of arbitrary quantum computation. Considered scheme of control not gate can be useful for the use in quantum free space communication lines with multiplexing by orbital angular momentum.

We emphasise our gratitude to the financial support of RFBR grant No. 18-29-20091.

\section{References}

1. T.D. Ladd, F. Jelezko, R. Laflamme, et al, Nature, 464, 45 (2010)

2. M. Mohseni, P. Read, H. Neven, et al, Nature, 543, 171 (2017)

3. E. Knill, R. Laflamme, and G. Milburn, Nature, 409, 46 (2001)

4. P. Kok, W.J. Munro, K. Nemoto, et al., Rev. Mod. Phys., 79, 35 (2007)

5. K. Nemoto, and W.J. Munro, Phys. Rev. Lett., 93, 250502 (2004)

6. G.M. D'Ariano, C. Macchiavello, and L. Maccone, Fortschr. Phys., 48, 573 (2000)

7. S.N. Andrianov, A.A. Kalachev, O.P. Shindyaev, and A.V. Shkalikov, Bulletin of the Russian Academy of Sciences: Physics, 83, 381 (2019)

8. D.F. Walls, and G.J. Milburn, (Quantum Optics Springer, Berlin, 2008)

9. H. Huang, Y. Ren, G. Xie, Y. Yan, Y. Yue, N. Ahmed, M.P.J. Lavery,M.J. Padgett, S. Dolinar, M. Tur, and A.E. Willner, Optics Letters, 39, 1689 (2014)

10. Z.-H. Zhu, L.-W. Sheng, Z.-W. Lv, W.-M. He, and W. Gao, Scientific Reports, 7, 40526 (2017)

11. M. Padgett, and L. Allen, Contemp. Phys. 41, 275 (2000)

12. C. Rotschild, S. Zommer, S. Moed, O. Hershcovitz, and S.G. Lipson, Applied Optics, 43, 2397 (2004) 